\title{
The Weinstein conjecture for planar contact structures in dimen- sion three
}

\author{
Casim Abbas, Kai Cieliebak ${ }^{\dagger}$ and Helmut Hofer ${ }^{\ddagger}$
}

\begin{abstract}
In this paper we describe a general strategy for approaching the Weinstein conjecture in dimension three. We apply this approach to prove the Weinstein conjecture for a new class of contact manifolds (planar contact manifolds). We also discuss how the present approach reduces the general Weinstein conjecture in dimension three to a compactness problem for the solution set of a first order elliptic PDE.
\end{abstract}

Mathematics Subject Classification (2000). 53D35, 37J45.

Keywords. Contact structure, open book, periodic orbit, holomorphic curve.

\section{Contents}

1 Introduction . . . . . . . . . . . . . . . . . 772

1.1 Versions of the Weinstein conjecture . . . . . . . . . . . . . 772

1.2 Generalized holomorphic curve equations . . . . . . . . . . . . . 773

1.3 Open book decompositions and the main result . . . . . . . . . . 774

2 Recollections on finite energy spheres . . . . . . . . . . . . . . 775

2.1 The Reeb flow near a periodic orbit . . . . . . . . . . . . . . . 777

2.2 Asymptotics near a puncture . . . . . . . . . . . . . . . . 777

2.3 Linear Fredholm theory . . . . . . . . . . . . . . . . . . . . . 778

2.4 Nonlinear Fredholm theory . . . . . . . . . . . . . . . . . . . . . . 780

2.5 Algebraic invariants . . . . . . . . . . . . . . . . 783

2.6 An implicit function theorem . . . . . . . . . . . . . . . . 784

2.7 Intersections . . . . . . . . . . . . . . . . . . 786

3 Proof of the main result . . . . . . . . . . . . . . . . 788

3.1 Finite energy foliations for Giroux forms . . . . . . . . . . . 788

3.2 A cobordism . . . . . . . . . . . . . . . . . . . . 790

${ }^{*}$ Research partially supported by a Michigan State University IRGP grant

${ }^{\dagger}$ Research partially supported by DFG Grant CI45/2-1

${ }^{\ddagger}$ Research partially supported by NSF Grant DMS-0102298 
3.3 A compactness statement . . . . . . . . . . . . . . . . 790

3.4 Conclusion . . . . . . . . . . . . . . . . . 791

\section{Introduction}

The following considerations are part of the program initiated in [9] and extended in [10] of proving the general Weinstein conjecture in dimension three. The key observation in [9] was the equivalence between the assertion of the Weinstein conjecture and the existence of a non-constant holomorphic curve for a suitable nonlinear Cauchy-Riemann type equation. As discussed in [10], this equivalence has its limitations. However, it was suggested that a suitable modification of the holomorphic curve equation should be the key to a proof of the general Weinstein conjecture in dimension 3. In the current joint work of the authors, the proof of the general Weinstein conjecture in dimension three has been reduced to a compactness question of certain moduli spaces for the generalized holomorphic curve equation. As we know from Giroux's work, any (co-oriented) contact structure is supported by an open book decomposition. In our approach, the compactness problems only arise if the pages of the open book decomposition are non-planar (i.e., of positive genus). If the pages are planar these difficulties do not arise. In this paper we describe our approach for this particular case.

1.1. Versions of the Weinstein conjecture. Before we give more details we start by providing the necessary background. Consider a closed three-manifold $M$ equipped with a contact structure $\xi$. In this paper we assume all contact structures to be cooriented, i.e., $\xi=\operatorname{ker}(\lambda)$ is defined by a contact 1 -form $\lambda$. We denote the associated Reeb vector field by $X_{\lambda}$. Recall that the (generalized) three-dimensional Weinstein conjecture states the following, see [20]:

Conjecture (A. Weinstein, 1978). Every Reeb vector field X on a closed threedimensional manifold $M$ admits a periodic orbit.

In fact, Weinstein added the hypothesis that the first cohomology group $H^{1}(M ; \mathbb{R})$ vanishes, but there is no indication that this additional hypothesis is needed. Moreover, Weinstein made his conjecture for Reeb vector fields on odd-dimensional manifolds of arbitrary dimensions. We point out at there are strong indications that in fact a stronger form of the Weinstein conjecture is true, which we again formulate in the three-dimensional case:

Strong version of the Weinstein conjecture. For every Reeb vector field $X$ on a closed three-dimensional manifold $M$ there exist finitely many periodic orbits $\left(x_{i}, T_{i}\right)$, $i=1, \ldots, n$, so that the first homology classes $\left[x_{1}\right], \ldots,\left[x_{n}\right]$ induced by the loops 
Vol. 80 (2005) The Weinstein conjecture for planar contact structures in dimension three 773

$x_{i}: \mathbb{R} /\left(T_{i} \mathbb{Z}\right) \rightarrow M$ sum up to $0:$

$$
\sum_{i=1}^{n}\left[x_{i}\right]=0 .
$$

Here the periods $T_{i}>0$ need not to be the minimal periods.

We will say that the (strong) Weinstein conjecture holds for a contact form $\lambda$ if the associated Reeb vector field satisfies the conclusion of the (strong) Weinstein conjecture.

1.2. Generalized holomorphic curve equations. We write $\pi: T M \rightarrow \xi$ for the projection along $X_{\lambda}$. Fix a complex structure $J$ on $\xi$ such that $d \lambda(\cdot, J \cdot)$ defines a positive definite metric on $\xi$. We will call such complex structures compatible (with $d \lambda$ ). Let us begin with an assertion reducing the Weinstein conjecture to the study of the following nonlinear first order elliptic system. The solutions of interest are 5-tuples $(S, j, \Gamma, \tilde{u}, \gamma)$ consisting of a closed Riemann surface $(S, j)$, a finite subset $\Gamma \subset S$, a proper map $\tilde{u}=(a, u): \dot{S} \rightarrow \mathbb{R} \times M$, where $\dot{S}=S \backslash \Gamma$, and a one-form $\gamma$ on $S$ so that

$$
\left.\begin{array}{l}
\pi \circ T u \circ j=J \circ \pi \circ T u \text { on } \dot{S}, \\
\left(u^{*} \lambda\right) \circ j=d a+\gamma \text { on } \dot{S}, \\
d \gamma=d(\gamma \circ j)=0 \text { on } S, \\
E(\tilde{u})<\infty .
\end{array}\right\}
$$

Here the energy $E(\tilde{u})$ is defined by

$$
E(\tilde{u})=\sup _{\varphi \in \Sigma} \int_{\dot{S}} \tilde{u}^{*} d(\varphi \lambda),
$$

where $\Sigma$ consists of all smooth maps $\varphi: \mathbb{R} \rightarrow[0,1]$ with $\varphi^{\prime}(s) \geq 0$ for all $s \in \mathbb{R}$. solutions of the system (1.1) energy curves.

The following theorem, which is an easy modification of a result by Hofer [9], [10], shows that the Weinstein conjecture is equivalent to an existence result for a generalized holomorphic curve (we restrict ourselves to the case of three dimensions in the following discussion):

Theorem 1.1. Let $(M, \lambda)$ be a closed three-dimensional manifold equipped with a contact form $\lambda$. Then the associated Reeb vector field has periodic orbits if and only if the associated PDE-problem (1.1) has a non-constant solution.

Note, however, that a nontrivial solution need not to have any puncture due to the harmonic perturbation $\gamma$. If the Riemann surface $(S, j)$ occurring in (1.1) is a sphere it follows immediately that $\gamma=0$, and the generalized equation reduces to the usual equation for punctured holomorphic curve in symplectizations. 
1.3. Open book decompositions and the main result. An open book decomposition of a closed 3-manifold $M$ is a pair ( $L$, pr) consisting of a fibered link $L \subset M$ (the binding) and a fibration pr: $M \backslash L \rightarrow S^{1}$ whose fibers $\operatorname{pr}^{-1}(t)$ (the pages) are the interiors of smooth compact embedded surfaces in $M$ bounded by $L$.

Definition 1.2. Following [7], we say that a contact structure $\xi$ on a closed 3-manifold $M$ is supported by an open book decomposition $(L, \mathrm{pr})$ if there exists a contact form $\lambda$ defining $\xi$ so that:

- The form $d \lambda$ induces an area form on each leaf $F$ of pr.

- The form $\lambda$ defines a volume form on $L$ inducing the orientation as boundary of $(F, d \lambda)$.

We will call $\lambda$ a Giroux form associated to $\left(L\right.$, pr) and denote such forms by $\lambda_{\text {Giroux }}$.

Remark 1.3. (1) The definition implies that each component of $L$ is a periodic orbit of the Reeb vector field associated to the Giroux form. We call the components of $L$ the binding orbits. Note that any Reeb orbit which is not a binding orbit hits any page in forward and backward time.

(2) A given Giroux form can be modified near the binding $L$ to have additional properties. For example, we can arrange that each binding orbit has a neighborhood isomorphic to that of a periodic orbit in the round sphere $S^{3}$. Alternatively, we can arrange that the binding orbits are nondegenerate elliptic periodic orbits (see [1]).

(3) Multiplying a Giroux form by some positive number we obtain another Giroux form.

Giroux's fundamental result is the following, see [7], [8].

Theorem 1.4. Any (co-orientable) contact structure on a closed 3-manifold $M$ is supported by an open book decomposition.

Definition 1.5. Let us call an open book decomposition planar if its pages have genus zero. Call a contact structure planar if it is supported by a planar open book decomposition.

The main result in this paper is the following:

Theorem 1.6 (Strong Weinstein Conjecture for Planar Contact Structures). Let $\xi$ be a planar contact structure on an oriented closed three-manifold $M$. Then the strong version of the Weinstein conjecture holds for any contact form defining $\xi$.

In view of this theorem and the results of [9], the Weinstein conjecture is now established for every contact form defining a contact structure $\xi$ on a closed oriented 3-manifold $M$ if at least one of the following conditions is met: 
Vol. 80 (2005) The Weinstein conjecture for planar contact structures in dimension three 775

(1) The contact structure $\xi$ is overtwisted ([9]).

(2) The second homotopy group of $M$ is nontrivial ([9]).

(3) The contact structure $\xi$ is planar (present paper).

Remark 1.7. (1) Recent progress in the understanding of contact three-manifolds, most notably an important result by Eliashberg [4], has led to serious advances in the study of the "planarity question". Indeed, in a recent paper [5], J. Etnyre shows that not all contact structures are planar. He also shows that every overtwisted contact structure is planar, so case (1) above is a consequence of case (3). The Weinstein conjecture remains open for tight contact forms on closed 3-manifolds with vanishing second homotopy group for which the underlying contact structure is not planar.

(2) It was pointed out by J. Etnyre that one can modify our proof in the planar case by putting on top of our construction Eliashberg's symplectic cobordism [4]. Then one can work with honest spheres rather than punctured spheres. The proof then has to make use of positivity of intersections, adjunction formula, self-intersection index, automatic transversality, and the compactness results for punctured holomorphic curves in [2]. Our arguments may be viewed as relative versions of these concepts.

The use of Eliashberg's cobordism would somewhat simplify the arguments in the planar case if one does not like to work with non-compact curves. However, an index calculation shows that Eliashberg's construction does not help to prove the Weinstein conjecture in non-planar cases, whereas our constructions are designed precisely for this case. The only problem at the moment is the lack of a compactness proof for the generalized equation (1.1), although we are making progress on this question.

Acknowledgement. The third author would like to thank Richard Siefring for helpful discussions on intersection questions which simplified some of our arguments.

\section{Recollections on finite energy spheres}

In this section we collect some facts about solutions of the PDE (1.1). Most of the results needed are scattered in the literature. Some of them need additional explanations and are further discussed. Throughout, $M$ is a closed oriented 3-manifold, $\xi$ is a contact structure defined by a contact form $\lambda, J$ is a compatible complex multiplication on $\xi$, and $\pi: T M \rightarrow \xi$ is the projection along the Reeb vector field $X_{\lambda}$. From now on, we will restrict ourselves to planar curves, i.e., the surface $S$ in (1.1) is diffeomorphic to the sphere. Then the harmonic form $\gamma$ vanishes and the PDE (1.1) reduces to

$$
\left.\begin{array}{l}
T u \circ j=J \circ \pi \circ T u, \\
\left(u^{*} \lambda\right) \circ j=d a, \\
E(\tilde{u})<\infty .
\end{array}\right\}
$$


A solution $(S, j, \Gamma, \tilde{u})$ of equation (2.1) is called a (special) finite energy sphere. Equation (2.1) can be written in a more concise form as follows. Associate to $J$ the almost complex structure $\tilde{J}$ on $\mathbb{R} \times M$ defined by

$$
\left.\tilde{J}\right|_{\xi}:=J: \xi \rightarrow \xi, \quad \tilde{J} \frac{\partial}{\partial r}:=X_{\lambda}, \quad \tilde{J} X_{\lambda}:=-\frac{\partial}{\partial r},
$$

where $r$ denotes the coordinate on $\mathbb{R}$. Note that $\tilde{J}$ is $\mathbb{R}$-invariant and compatible with the symplectic form $d\left(e^{r} \lambda\right)$ in the sense that $d\left(r^{r} \lambda\right)(\cdot, \tilde{J} \cdot)$ defines a Riemannian metric. Then equation (2.1) is equivalent to

$$
T \tilde{u} \circ j=\tilde{J} \circ T \tilde{u}, \quad E(\tilde{u})<\infty .
$$

We also need to consider a generalization of equation (2.1). Let $\lambda^{+}, \lambda^{-}$be two contact forms defining the same contact structure $\xi$ such that

$$
\lambda^{+}=f^{+} \cdot \lambda^{-}
$$

for a function $f^{+}>1$ on $M$. Pick a positive function $f$ on $\mathbb{R} \times M$ and a constant $R>0$ such that $\frac{\partial f}{\partial r} \geq 0$ and

$$
f(r, x)= \begin{cases}f^{+}(x) & \text { for } r \geq R, \\ 1 & \text { for } r \leq-R .\end{cases}
$$

Note that $\omega_{f}:=d\left(e^{r} f \lambda^{-}\right)$is a symplectic form on $\mathbb{R} \times M$. Let $\tilde{J}$ be a compatible almost complex structure on $\mathbb{R} \times M$. This means that $\omega_{f}(\cdot, \tilde{J} \cdot)$ defines a Riemannian metric. Moreover, we assume that

$$
\tilde{J}= \begin{cases}\tilde{J}^{+} & \text {on }[R, \infty) \times M, \\ \tilde{J}^{-} & \text {on }(-\infty,-R] \times M,\end{cases}
$$

where $\tilde{J}^{ \pm}$are the $\mathbb{R}$-invariant almost complex structures associated to complex multiplications $J^{ \pm}: \xi \rightarrow \xi$ compatible with $\lambda^{ \pm}$. We now study smooth maps $\tilde{u}: \dot{S} \rightarrow$ $\mathbb{R} \times M$ satisfying

$$
T \tilde{u} \circ j=\tilde{J} \circ T \tilde{u}, \quad E(\tilde{u})<\infty .
$$

Here the energy $E(\tilde{u})$ is defined by

$$
E(\tilde{u})=\sup _{\varphi \in \Sigma} \int_{\dot{S}} \tilde{u}^{*} d\left(\varphi f \lambda^{-}\right),
$$

with $\Sigma$ as before. We call solutions of equation (2.2) generalized finite energy spheres. 
Vol. 80 (2005) The Weinstein conjecture for planar contact structures in dimension three 777

2.1. The Reeb flow near a periodic orbit. Let $x$ be a periodic Reeb orbit on $(M, \lambda)$ of period $T$. Denote by $\phi_{t}: M \rightarrow M$ the Reeb flow, thus $\phi_{T}(x(0))=x(0)$. The linearized Reeb flow along $x$ gives rise to a family of linear maps $\Phi_{t}: \xi_{x(0)} \rightarrow \xi_{x(t)}$ which preserve the symplectic form $\left.d \lambda\right|_{\xi}$. We call $x$ nondegenerate if $\Phi_{T}: \xi_{x(0)} \rightarrow \xi_{x(0)}$ does not have 1 in its spectrum. Then two cases can occur. Either both eigenvalues are real, then we call $x$ hyperbolic, or both are non-real, then $x$ is called elliptic.

Closely related to the linearized Reeb flow is the asymptotic operator along $x$

$$
A \eta:=-J(x)\left(\nabla_{t} \eta-\nabla_{\eta} X_{\lambda}\right)
$$

acting on sections $\eta(t)=\eta(t+T)$ of the bundle $x^{*} \xi$. Its kernel corresponds to eigenvectors of $\Phi_{T}$ with eigenvalue 1, so in the nondegenerate case the kernel is trivial. Moreover, eigenfields of $A$ have no zeroes. Fix a trivialization of the bundle $x^{*} \xi$. In this trivialization each eigenfield of the self-adjoint operator $A$ has a winding number which depends only on the eigenvalue, see [11] for details. The winding number increases with the eigenvalue, and each winding number occurs for precisely two eigenvalues (counted with multiplicities). Denote by $\alpha(x)$ the winding number corresponding to the largest negative eigenvalue. If $x$ is nondegenerate and elliptic both eigenvalues with winding number $\alpha(x)$ are negative and the Conley-Zehnder index of $x$ (in the given trivialization, see [11]) is given by

$$
\mu(x)=2 \alpha(x)+1 .
$$

We will also need a weighted version of this relation. For a weight $\delta<0$ which is not an eigenvalue define the weighted Conley-Zehnder index by

$$
\mu_{\mathrm{w}}(x):=2 \alpha_{\mathrm{w}}(x)+1,
$$

where $\alpha_{\mathrm{W}}(x)$ is the winding number corresponding to the largest eigenvalue $<\delta$. Note that $\alpha_{w}(x)$ and $\mu_{\mathrm{w}}(x)$ are the winding number corresponding to the largest negative eigenvalue, respectively Conley-Zehnder index, of the weighted asymptotic operator $A_{\mathrm{w}}:=A-\delta$.

2.2. Asymptotics near a puncture. Next we describe the behavior of solutions of (2.1) near a positive puncture. The reference for this section is [14]. Let $x$ be a periodic Reeb orbit of period $T>0$. Denote by $\tau>0$ the minimal period and by $k \in \mathbb{N}$ its covering number, so that $T=k \tau$. In suitable local coordinates in a tubular neighborhood $U$ of $x$ the contact form is given by

$$
\lambda=f(d \vartheta+x d y)
$$

where $(\vartheta, x, y) \in S^{1} \times \mathbb{R}^{2}$ with $S^{1}=\mathbb{R} / \mathbb{Z}$. Here the periodic orbit $x$ corresponds to $t \mapsto(k t, 0,0) \in S^{1} \times \mathbb{R}^{2}$, and $f>0$ is a function satisfying $f(\vartheta, 0,0)=\tau$ and $d f(\vartheta, 0,0)=0$. 
Let $\tilde{u}=(a, u):[0, \infty) \times S^{1} \rightarrow \mathbb{R} \times M$ be a solution of (2.1) such that $u(s, \cdot) \rightarrow x$ and $a(s, \cdot) \rightarrow+\infty$ as $s \rightarrow \infty$. After replacing $[0, \infty) \times S^{1}$ by $[R, \infty) \times S^{1}$ for a sufficiently large $R$, we may assume that the image of $u$ is contained in a neighbourhood $U \subset S^{1} \times \mathbb{R}^{2}$ above. Hence we can write

$$
\tilde{u}(s, t)=(a(s, t), \vartheta(s, t), z(s, t))
$$

in the coordinates above, with $z=(x, y) \in \mathbb{R}^{2}$. The following asymptotic behaviour was established in [14].

Theorem 2.1. Suppose that $x$ is nondegenerate of period $T$ and covering number $k$. Then there exist constants $a_{0}, \vartheta_{0} \in \mathbb{R}$ and $d>0$ such that

$$
\begin{aligned}
\left|\partial^{\beta}\left[a(s, t)-T s-a_{0}\right]\right| & \leq C_{\beta} e^{-d s}, \\
\left|\partial^{\beta}\left[\vartheta(s, t)-k t-\vartheta_{0}\right]\right| & \leq C_{\beta} e^{-d s}
\end{aligned}
$$

for all multi-indices $\beta$, with constants $C_{\beta}$ depending on $\beta$. Moreover, if the z-part does not vanish identically we have the asymptotic formula for the transversal approach to $x(t)$ :

$$
z(s, t)=e^{\int_{s_{0}}^{s} \lambda(\sigma) d \sigma}[e(t)+r(s, t)] \in \mathbb{R}^{2},
$$

where $\partial^{\beta} r(s, t) \rightarrow 0$ as $s \rightarrow \infty$, uniformly in t for all derivatives. Here $\lambda:\left[s_{0}, \infty\right) \rightarrow$ $\mathbb{R}$ is a smooth function satisfying

$$
\lambda(s) \rightarrow \lambda<0 \text { as } s \rightarrow+\infty,
$$

where $\lambda<0$ is an eigenvalue of the asymptotic operator A along $x$ defined in the previous section and $e(t)=e(t+1) \neq 0$ is an eigenfield to $\lambda$.

In particular, this implies that $u: \dot{S} \rightarrow M$ admits a continuous extension $\bar{u}$ to the circle compactification $\bar{S}$ of its domain $\dot{S}$. The behaviour near a negative puncture (at which $a \rightarrow-\infty$ ) is similar. In the following we will only need positive punctures. Note that equation 2.2 agrees with equation (2.1) for $|r| \geq R$, so Theorem 2.1 also applies to generalized finite energy spheres.

2.3. Linear Fredholm theory. Following [17], we introduce a special class of linear Fredholm operators over a punctured Riemann sphere $(\dot{S}, j)$. They act on sections of a trivial complex line bundle $V=\dot{S} \times \mathbb{R}^{2}$ with fibrewise complex structure $i=i(z)$, $z \in \dot{S}$. Denote by $\mathcal{A}_{0} \rightarrow \dot{S}$ the bundle of complex antilinear bundle homomorphisms $T \dot{S} \rightarrow V$. Let $C$ be a smooth section in the bundle $\operatorname{Hom}_{\mathbb{R}}\left(V, \mathcal{A}_{0}\right) \rightarrow \dot{S}$ of real bundle homomorphisms $V \rightarrow \mathcal{A}_{0}$. We call $C$ admissible if at every puncture the following holds. (We assume all punctures to be positive, although for this subsection this makes no difference). Let $(s, t)$ be polar coordinates such that $s \rightarrow \infty$ at the 
Vol. 80 (2005) The Weinstein conjecture for planar contact structures in dimension three 779

puncture. Then there exist smooth loops of complex structures $i^{+}(t)$ on $\mathbb{R}^{2}$ and $2 \times 2$-matrices $C^{+}(t)$ such that

$$
i(s, t) \rightarrow i^{+}(t), \quad C(s, t) \cdot \frac{\partial}{\partial s} \rightarrow C^{+}(t)
$$

in $C^{\infty}$ as $s \rightarrow \infty$. The matrices $C^{+}(t)$ are symmetric with respect to the metrics $\omega\left(\cdot, i^{+}(t) \cdot\right)$, where $\omega$ is the standard symplectic form on $\mathbb{R}^{2}$. Moreover, we require that the asymptotic operator

$$
A^{+} \eta:=-i^{+}(t) \frac{\partial \eta}{\partial t}-C^{+}(t) \eta
$$

acting on smooth functions $\eta: S^{1} \rightarrow \mathbb{R}^{2}$ has trivial kernel. Thus the equation $A^{+} \eta=$ 0 defines a path of symplectic $2 \times 2$-matrices $\Phi_{t}$ such that $\Phi_{0}=\mathrm{Id}$ and $\Phi_{1}$ does not have 1 in its spectrum. Denote by $\mu^{+}$the Conley-Zehnder index of this path.

We associate to an admissible $C$ the operator $L_{C}: \Omega^{0}(V) \rightarrow \Omega^{0}\left(\mathcal{A}_{0}\right)$

$$
L_{C} v:=T v+i \circ T v \circ j+C v
$$

acting on sections of the bundle $V \rightarrow \dot{S}$. Let $\# \Gamma$ be the number of (positive) punctures of $\dot{S}$ and

$$
\mu\left(L_{C}\right):=\sum_{j} \mu_{j}^{+}
$$

the sum of the Conley-Zehnder indices at the punctures. The following result was proved in [17].

Proposition 2.2. The operator $L_{C}$ associated to an admissible $C$ defines a Fredholm operator $L_{C}: E \rightarrow F$ between suitable Sobolev (or Hölder) completions of $\Omega^{0}(V)$ and $\Omega^{0}\left(\mathcal{A}_{0}\right)$ of index

$$
\operatorname{ind}\left(L_{C}\right)=\mu\left(L_{C}\right)+2-\# \Gamma .
$$

The arguments in [14] show that elements in the kernel of $L_{C}$ have asymptotics at a puncture analogous to the $\xi$-component $z$ in Theorem 2.1.

Corollary 2.3. A nontrivial element $v$ in the kernel of $L_{C}$ has the following asymptotic behaviour in polar coordinates near a puncture:

$$
v(s, t)=e^{\int_{s_{0}}^{s} \lambda(\sigma) d \sigma}[e(t)+r(s, t)] \in \mathbb{R}^{2},
$$

where $\partial^{\beta} r(s, t) \rightarrow 0$ as $s \rightarrow \infty$, uniformly in t for all derivatives. Here $\lambda:\left[s_{0}, \infty\right) \rightarrow$ $\mathbb{R}$ is a smooth function satisfying

$$
\lambda(s) \rightarrow \lambda<0 \text { as } s \rightarrow+\infty,
$$

where $\lambda<0$ is an eigenvalue of the asymptotic operator $A^{+}$at the puncture and $e(t)=e(t+1) \neq 0$ is an eigenfield to $\lambda$. 
Let us discuss the effect of exponential weights. By Corollary 2.3, a nontrivial element in the kernel of $L_{C}$ approaches zero at the $j$-th puncture with an exponential rate given by an eigenvalue $\lambda_{j}<0$ of the asymptotic operator $A_{j}^{+}$. For weights $\lambda_{j}<\delta_{j}<0$ that are not eigenvalues, denote by $E_{\mathrm{w}}, F_{\mathrm{w}}$ the weighted Sobolev spaces of sections converging to zero at the punctures with exponential rates

$\delta_{j}$ or faster. Thus $E_{\mathrm{w}}$ is the space of sections $\eta$ in $V \rightarrow \dot{S}$ such that $\eta_{\mathrm{w}} \in E$, where $\eta_{\mathrm{w}}$ is defined by multiplying $\eta$ by a positive smooth function which agrees with $e^{-\delta_{j} s}$ near the $j$-th puncture, and $F_{\mathrm{w}}$ similarly. Define the weighted Fredholm index $\operatorname{ind}_{\mathrm{w}}\left(L_{C}\right)$ as the index of the linear Fredholm operator $L_{C}: E_{\mathrm{w}} \rightarrow F_{\mathrm{w}}$. Note that $\eta \rightarrow \eta_{\mathrm{w}}$ defines an isomorphism $E_{\mathrm{w}} \rightarrow E$ (and similarly for $F$ ) which conjugates the operator $L_{C}: E_{\mathrm{w}} \rightarrow F_{\mathrm{w}}$ to the operator $L_{C_{\mathrm{w}}}: E \rightarrow F$ associated to an admissible $C_{\mathrm{w}}$. A simple computation shows that the asymptotic operators of $C_{\mathrm{w}}$ are precisely the weighted asymptotic operators (cf. Section 2.1) at the punctures. Hence by Proposition 2.2,

$$
\operatorname{ind}_{\mathrm{w}}\left(L_{C}\right)=\operatorname{ind}\left(L_{C_{\mathrm{w}}}\right)=\mu_{\mathrm{w}}\left(L_{C}\right)+2-\# \Gamma,
$$

where $\mu_{\mathrm{w}}\left(L_{C}\right)$ is the sum of the weighted Conley-Zehnder indices at the punctures.

Next consider a nontrivial element $v$ in the kernel of $L_{C}$. By Corollary 2.3, it converges to zero at the $j$-th puncture from the direction of some eigenvector $e_{j}$ of the asymptotic operator $A_{j}^{+}$. Denote the winding number of $e_{j}$ by $w_{j}^{+}$and define the winding number of $v$ by

$$
\operatorname{wind}(v):=\sum w_{j}^{+}
$$

The asymptotics of $v$ and the similarity principle imply (cf. [17])

Lemma 2.4. Let $v$ be a nontrivial element in the kernel of $L_{C}$. Then $v$ has only finitely many zeroes, each zero has positive multiplicity, and their algebraic sum equals wind $(v)$.

2.4. Nonlinear Fredholm theory. Next we recall the Fredholm theory for equation (2.2). The basic references are [17] for the embedded case (which is all we need), and [3] for the general case. Let $\tilde{u}=(a, u): \dot{S} \rightarrow R \times M$ be a generalized finite energy sphere with asymptotic orbits $x_{j}$. From now on we assume that all the punctures are positive (i.e., $a \rightarrow+\infty$ ) and all the asymptotic orbits are distinct, simple and nondegenerate elliptic. Denote by $\mu_{j}$ and $\alpha_{j}$ their Conley-Zehnder indices, respectively winding numbers of the largest negative eigenvalue, with respect to trivializations induced by a trivialization of $u^{*} \xi$ over $\dot{S}$. So we have

$$
\mu_{j}=2 \alpha_{j}+1 .
$$

Denote by $\mu(\tilde{u}):=\sum \mu_{j}$ the Conley-Zehnder index of $\tilde{u}$ and by $\# \Gamma$ the number of (positive) punctures of $\dot{S}$. 
Vol. 80 (2005) The Weinstein conjecture for planar contact structures in dimension three 781

Denote by $\mathcal{M}$ the moduli space of solutions of equation (2.2) with \#Г positive punctures and asymptotic orbits $x_{j}$.

The space $\mathcal{M}$ can be described as the zero set of the nonlinear Cauchy-Riemann operator defined by (2.2) on a suitable Banach manifold of maps $\dot{S} \rightarrow \mathbb{R} \times M$ times the moduli space $\mathcal{M}_{0, \# \Gamma}$ of $\# \Gamma$ points on the sphere. Its linearization at $\tilde{u}$ is a linear Fredholm operator $D: E \times T \mathcal{M}_{0, \# \Gamma} \rightarrow F$ between Banach spaces. Here $E$ and $F$ are suitable Sobolev completions of the space of sections, respectively $(0,1)$-forms, in the pullback bundle $\tilde{u}^{*} T(\mathbb{R} \times M)$. According to [17] for embeddings $\tilde{u}$, and [3] in general, the Fredholm index of $D$ is given by

$$
\operatorname{ind}(\tilde{u})=\mu(\tilde{u})-2+\# \Gamma \text {. }
$$

If $\tilde{u}$ is an embedding there is an alternative description developed in [17]. Write nearby curves as graphs of sections in the complex normal bundle $N \rightarrow \dot{S}$ to $C=\tilde{u}(\dot{S})$ in $\mathbb{R} \times M$. Equation (2.2) translates into a Monge-Ampere type equation for sections of $N$ whose linearization $D^{N}$ at the zero-section is the projection of $D$ onto $N$. Set $\mu^{N}(\tilde{u}):=\sum \mu_{j}^{N}$, where the normal Conley-Zehnder indices

$$
\mu_{j}^{N}=2 \alpha_{j}^{N}+1
$$

are computed with respect to trivializations induced by a trivialization of the normal bundle $N \rightarrow \dot{S}$. Note that in view of the asymptotics (Theorem 2.1) the bundles $u^{*} \xi$ and $N$ agree near the punctures. Comparing these bundles over $\dot{S}$ yields the following relation between the Conley-Zehnder index and the normal Conley-Zehnder index, see [14]:

$$
\mu(\tilde{u})=\mu^{N}(\tilde{u})+4-2 \sharp \Gamma .
$$

It allows us to express the index of $\tilde{u}$ in terms of the normal Conley-Zehnder index:

$$
\operatorname{ind}(\tilde{u})=\mu^{N}(\tilde{u})+2-\# \Gamma .
$$

On the other hand, the operator $D^{N}$ is an admissible operator of the form considered in Section 2.3 (see the proof of Lemma 2.5 below), and by Proposition 2.2 its index is given by the right-hand side of equation (2.7). This must of course be the case because $D$ and $D^{N}$ both describe nearby solutions of the same equation (2.2). The following lemma is implicit in [17].

Lemma 2.5. Let $\tilde{u}$ be an embedded solution of (2.2) with only positive punctures asymptotic to elliptic Reeb orbits $x_{j}$. Let $v$ be a section in the normal bundle $N \rightarrow \dot{S}$ whose graph describes a solution of (2.2) near $\tilde{u}$. Then $v$ satisfies a linear equation $L_{\hat{C}} v=0$, where $\hat{C}$ is an admissible operator on $N$ in the sense of Section 2.3. Moreover, the asymptotic operators of $\hat{C}$ at the punctures agree with the asymptotic operators at the periodic orbits $x_{j}$ as in (2.3). 
Proof. Let us sketch the proof. Denote coordinates on $\dot{S}$ by $z$ and on $\mathbb{R}^{2}$ by $x$. Pick a trivialization $N \cong \dot{S} \times \mathbb{R}^{2}$ as provided by Theorem 4.7 in [17]. Write the almost complex structure in this trivialization as

$$
\tilde{J}=\left(\begin{array}{cc}
j & \tilde{\Delta} \\
\Delta & i
\end{array}\right): T \dot{S} \times \mathbb{R}^{2} \rightarrow T \dot{S} \times \mathbb{R}^{2}
$$

where the components of $\tilde{J}$ depend smoothly on $(z, x)$. Since the zero section $\dot{S} \times\{0\}$ is $\tilde{J}$-holomorphic, we have

$$
\tilde{J}(z, 0)=\left(\begin{array}{cc}
j(z) & \tilde{\Delta}(z) \\
0 & i(z)
\end{array}\right)
$$

for complex structures $j$ on $\dot{S}$ and $i$ on $\mathbb{R}^{2}$ and homomorphisms $\tilde{\Delta}(z): \mathbb{R}^{2} \rightarrow T_{z} \dot{S}$. According to Section 5 in [17], the section $C$ in the bundle $\operatorname{Hom}_{\mathbb{R}}\left(N, \mathcal{A}_{0}\right) \rightarrow \dot{S}$ defined by

$$
C(z) h:=\left(\frac{\partial \Delta}{\partial x}(z, 0) h\right) \circ j(z, 0), \quad h \in \mathbb{R}^{2},
$$

is admissible and the corresponding operator

$$
w \mapsto L_{C} w=T w+i \circ T w \circ j+C w
$$

agrees with the normal linearized Cauchy-Riemann operator $D^{N}$ at $\tilde{u}$. In particular, the asymptotic operators of $C$ at the punctures agree with the asymptotic operators at the periodic orbits $x_{j}$.

By hypothesis, the graph $\operatorname{gr}(v)$ of $v$ satisfies the equation

$$
T \operatorname{gr}(v)+\tilde{J}(\operatorname{gr}(v)) \circ T \operatorname{gr}(v) \circ \hat{\jmath}=0
$$

for some complex structure $\hat{\jmath}$ on $\dot{S}$. The $\dot{S}$-component of this equation yields $\hat{\jmath}(z)=$ $j(z)+\Delta(z, v) \circ T v$; its $\mathbb{R}^{2}$-component is

$$
T v+i(z, v) \circ T v \circ \hat{\jmath}+\Delta(z, v) \circ \hat{\jmath}=0 .
$$

Define the complex structures $\hat{\imath}(z):=i(z, v(z))$ on $\mathbb{R}^{2}$ and the section $\hat{C}$ in the bundle $\operatorname{Hom}_{\mathbb{R}}\left(N, \mathcal{A}_{0}\right) \rightarrow \dot{S}$ by

$$
\hat{C}(z) h:=\int_{0}^{1}\left(\frac{\partial \Delta}{\partial x}(z, \tau v(z)) h\right) \circ \hat{\jmath} d \tau, \quad h \in \mathbb{R}^{2} .
$$

Then the equation for $v$ can be viewed as a linear equation as in Section 2.3,

$$
L_{\hat{C}} v=T v+\hat{\imath} \circ T v \circ \hat{\jmath}+\hat{C} v=0 .
$$

Note that near a puncture $v(s, t) \rightarrow 0$ in $C^{\infty}$ as $s \rightarrow \infty$, so $\hat{C}$ approaches $C$ at the punctures. This implies that $\hat{C}$ is admissible with the same asymptotic operators as $C$ and the lemma follows. 
Vol. 80 (2005) The Weinstein conjecture for planar contact structures in dimension three 783

Finally, let us discuss the effect of exponential weights. By Theorem 2.1, $u(s, t)$ approaches $x_{j}(t)$ normally with an exponential rate given by an eigenvalue $\lambda_{j}<0$ of the asymptotic operator at $x_{j}$. For weights $\lambda_{j}<\delta_{j}<0$ that are not eigenvalues, denote by $\mathcal{M}_{\mathrm{w}}$ the space of solutions in $\mathcal{M}$ which normally approach the $x_{j}$ at the (positive) punctures with an exponential rate $\delta_{j}$ or faster. By construction, the solution $\tilde{u}$ belongs to $\mathcal{M}_{\mathrm{w}}$. Define the weighted Fredholm index $\operatorname{ind}_{\mathrm{w}}(\tilde{u})$ as the index of the linear Fredholm operator $D: E_{\mathrm{w}} \rightarrow F_{\mathrm{w}}$ between suitable weighted Sobolov spaces describing nearby solutions in $\mathcal{M}_{\mathrm{w}}$. An argument as in Section 2.3 shows (see [17], Section 6)

$$
\operatorname{ind}_{\mathrm{w}}(\tilde{u})=\operatorname{ind}\left(D_{\mathrm{w}}\right)=\mu_{\mathrm{w}}(\tilde{u})-2+\# \Gamma .
$$

Similarly, the relations (2.7) and (2.6) carry over to the weighted case.

2.5. Algebraic invariants. In this section we use the algebraic invariants from [12] to single out a 2-parameter family of solutions by putting suitable exponential weights. Let $\tilde{u}=(a, u): \dot{S} \rightarrow R \times M$ be a (special or generalized) finite energy sphere with asymptotic orbits $x_{j}$. As in the previous section, suppose that all the punctures are positive and all the asymptotic orbits are nondegenerate and elliptic with ConleyZehnder indices $\mu_{j}=2 \alpha_{j}+1$. By Theorem 2.1, the solution $u$ approaches $x_{j}$ from the direction of some eigenvector $e_{j}$ of the asymptotic operator. Denote the winding number of $e_{j}$ with respect to a trivialization of $u^{*} \xi$ by $w_{j}$ and define the asymptotic winding number of $\tilde{u}$ by

$$
\operatorname{wind}_{\infty}(\tilde{u}):=\sum w_{j}
$$

Since $u$ approaches $x_{j}$ from the direction of an eigenvector to a negative eigenvalue at a positive puncture, we have

$$
w_{j} \leq \alpha_{j}
$$

Let $\tau:=\sum\left(\alpha_{j}-w_{j}\right)$ be the difference between the actual winding numbers at the punctures and the maximal possible ones.

Now assume that $\tilde{u}$ is a special finite energy sphere. Then, according to [12], the section $\pi \circ T u$ of the bundle $\operatorname{Hom}_{\mathbb{C}}\left(T \dot{S}, u^{*} \xi\right)$ satisfies a linear Cauchy-Riemann type equation as in Section 2.3 (this is not true for generalized finite energy spheres). The $z$-part in Theorem 2.1 cannot vanish identically because if it did then $\tilde{u}$ would be a covering of the cylinder over $x_{j}$ and thus have negative punctures (Theorem 6.11. in [12]). By the similarity principle, $\pi \circ T u$ can only vanish in finitely many points. The winding number $\operatorname{wind}_{\pi}(\tilde{u})$ is then defined as the sum of the indices of the zeroes of this section. It is a nonnegative integer which measures how often $u$ is tangent to the Reeb vector field and is related to the asymptotic winding number by the formula (see [12])

$$
\operatorname{wind}_{\pi}(\tilde{u})=\operatorname{wind}_{\infty}(\tilde{u})-2+\# \Gamma \text {. }
$$


Combining formulae (2.9) and (2.5), we find

$$
\begin{aligned}
2 \tau & =2 \sum\left(\alpha_{j}-w_{j}\right) \\
& =\sum\left(2 \alpha_{j}+1\right)-\# \Gamma-2 \operatorname{wind}_{\infty}(\tilde{u}) \\
& =\mu(\tilde{u})-\# \Gamma-2 \operatorname{wind}_{\infty}(\tilde{u}) \\
& =\mu(\tilde{u})-2 \operatorname{wind}_{\pi}(\tilde{u})-4+\# \Gamma \\
& =\operatorname{ind}(\tilde{u})-2-2 \operatorname{wind}_{\pi}(\tilde{u}) .
\end{aligned}
$$

Now pick weights $\delta_{j}<0$ just above the larger eigenvalue corresponding to $w_{j}$. Denote by $\alpha_{j}^{w}$ the winding number corresponding to the largest eigenvalue smaller than $\delta_{j}<0$. Then $\alpha_{j}^{w}=w_{j}$ and the sum $\mu_{\mathrm{w}}(\tilde{u})$ of the corresponding weighted Conley-Zehnder indices satisfies

$$
\begin{aligned}
\mu_{w}(\tilde{u}) & =2 \sum \alpha_{j}^{w}+\# \Gamma \\
& =2 \sum w_{j}+\# \Gamma \\
& =-2 \tau+2 \sum \alpha_{j}+\# \Gamma \\
& =\mu(\tilde{u})-2 \tau .
\end{aligned}
$$

The weighted Fredholm index (2.4) becomes

$$
\begin{aligned}
\operatorname{ind}_{\mathrm{w}}(\tilde{u}) & =\mu_{\mathrm{w}}(\tilde{u})-2+\# \Gamma \\
& =\operatorname{ind}(\tilde{u})-2 \tau \\
& =2+2 \operatorname{wind}_{\pi}(\tilde{u}) .
\end{aligned}
$$

In particular, if $u$ is embedded and transverse to the Reeb vector field the winding

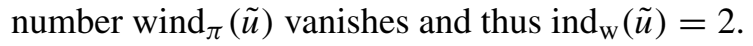

So we have shown

Lemma 2.6. Let $\tilde{u}=(a, u): \dot{S} \rightarrow \mathbb{R} \times M$ be a special finite energy sphere having only positive punctures. Suppose that all the asymptotic orbits are nondegenerate elliptic and that $u$ is embedded and transverse to the Reeb flow. Then we can introduce exponential weights at the punctures such that $\tilde{u}$ belongs to the space $\mathcal{M}_{\mathrm{w}}$ of solutions with these weights, and the Fredholm index of $\tilde{u}$ with these weights satisfies $\operatorname{ind}_{\mathrm{w}}(\tilde{u})=2$.

2.6. An implicit function theorem. Now fix a collection of distinct nondegenerate simple elliptic orbits $x_{j}$ and weights $\delta_{j}<0$. Consider the space $\mathcal{M}_{\mathrm{w}}$ of generalized finite energy spheres with positive punctures asymptotic to the $x_{j}$ with exponential decay $\delta_{j}$ or faster. The following result describes the local structure of the set $\mathcal{M}_{\mathrm{w}}$. 
Vol. 80 (2005) The Weinstein conjecture for planar contact structures in dimension three 785

Theorem 2.7. Assume that $C=\tilde{u}(\dot{S}) \in \mathcal{M}_{\mathrm{w}}$ is embedded and has weighted Fredholm

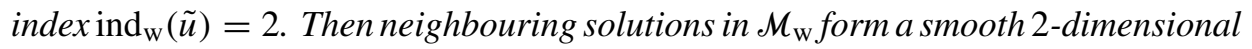
family of mutually disjoint embedded curves.

Proof. The argument is similar to that given in the proof of Theorem 2.7 in [18]. As in Section 2.4, we write neighbouring solutions in $\mathcal{M}_{\mathrm{w}}$ as graphs of sections of the complex normal bundle to $C$. They satisfy a Monge-Ampere type equation whose linearization $D^{N}: E_{\mathrm{w}} \rightarrow F_{\mathrm{w}}$ at the zero section is a Cauchy-Riemann type operator as in Section 2.3 between suitable Sobolev spaces with weights $\delta_{j}$.

Consider a nontrivial element $h$ in the kernel of $D^{N}$. By Corollary 2.3, $h$ approaches zero at the $j$-th puncture exponentially from a direction $e_{j}(t)$, where $e_{j}$ is an eigenfield of the asymptotic operator at $x_{j}$. According to Lemma $2.4, h$ has a winding number

$$
\operatorname{wind}(h)=\sum w_{j},
$$

where $w_{j}$ is the winding number of $e_{j}: S^{1} \rightarrow \mathbb{R}^{2} \backslash\{0\}$ with respect to a trivialization of the normal bundle $N$. Let

$$
\mu_{\mathrm{w}}^{N}\left(x_{j}\right)=2 \cdot \alpha_{\mathrm{w}}^{N}\left(x_{j}\right)+1
$$

be the weighted normal Conley-Zehnder index at $x_{j}$, where $\alpha_{\mathrm{w}}^{N}\left(x_{j}\right)$ is the maximal winding number of an eigenfield of the asymptotic operator $A_{j}$ associated to an eigenvalue $<\delta_{j}$. Since $h$ belongs to the Sobolev space with weights $\delta_{j}$, we have

$$
w_{j} \leq \alpha_{\mathrm{w}}^{N}\left(x_{j}\right) .
$$

With $\mu_{\mathrm{w}}^{N}(\tilde{u}):=\sum \mu_{\mathrm{w}}^{N}\left(x_{j}\right)$ and the weighted version of formula (2.7), this implies

$$
\begin{aligned}
2 \operatorname{wind}(h) & =\sum_{j} 2 w_{j} \\
& \leq \sum_{j}\left[2 \alpha_{\mathrm{w}}^{N}\left(x_{j}\right)+1\right]-\sharp \Gamma \\
& =\mu_{\mathrm{w}}^{N}(\tilde{u})-\sharp \Gamma \\
& =\operatorname{ind}_{\mathrm{w}}(\tilde{u})-2 \\
& =0 .
\end{aligned}
$$

This shows that nontrivial elements in the kernel of $D^{N}$ are nowhere vanishing. It follows that the kernel can be at most two-dimensional, since otherwise we could construct a nontrivial element in the kernel with a zero. Since $\operatorname{ind}_{\mathrm{w}}(\tilde{u})=2$, we conclude that the operator $D^{N}$ is surjective. Thus $\mathcal{M}_{\mathrm{w}}$ is a smooth 2-dimensional manifold near $C$. 
It remains to prove that neighbouring elements $C^{\prime} \neq C$ in $\mathcal{M}_{\mathrm{w}}$ do not intersect $C$. As in Section 2.4, describe $C^{\prime}$ as the graph of a nonvanishing section $v$ of the normal bundle to $C$. By Lemma 2.5, $v$ satisfies a linear Cauchy-Riemann type equation $L_{\hat{C}} v=0$, where the admissible section $\hat{C}$ has the same asymptotics as $D^{N}$. Hence the winding number of $v$ satisfies

$$
\operatorname{wind}(v)=\sum w_{j} .
$$

Now the computation above shows wind $(v)=0$. Hence $v$ has no zeroes, which precisely means that its graph does not intersect $C$.

Definition 2.8. For $C \in \mathcal{M}_{\mathrm{w}}$ we denote by $a(C) \in \mathbb{R}$ the minimum of the $\mathbb{R}$-value of the projection $C \rightarrow \mathbb{R}$.

Theorem 2.7 has the following immediate corollary.

Corollary 2.9. For $C \in \mathcal{M}_{\mathrm{w}}$ as in Theorem 2.7 there exists a $C^{\prime} \in \mathcal{M}_{\mathrm{w}}$ with

$$
a\left(C^{\prime}\right)<a(C) .
$$

2.7. Intersections. Consider a connected component $\mathcal{M}_{\mathrm{w}}^{0}$ of $\mathcal{M}_{\mathrm{w}}$ containing an embedded solution $C_{0}$ of index 2. By Theorem 2.7 and positivity of intersections (see [6]), this implies that $\mathcal{M}_{\mathrm{w}}^{0}$ is a smooth 2-dimensional manifold and all elements in $\mathcal{M}_{\mathrm{w}}^{0}$ are embedded. Moreover, nearby distinct elements in $\mathcal{M}_{\mathrm{w}}^{0}$ are disjoint. The following result shows that any two (not necessarily nearby) elements are either identical or disjoint.

Proposition 2.10. Two elements $C, C^{\prime}$ in $\mathcal{M}_{\mathrm{w}}^{0}$ are either identical or disjoint.

Proof. First note that two distinct $C, C^{\prime} \in \mathcal{M}_{\mathrm{w}}^{0}$ intersect only in finitely many points. To see this, write $C^{\prime}$ near the $j$-th puncture as the graph of a nontrivial section $v_{j}$ in the normal bundle to $C$. By Lemma 2.5 and Lemma 2.4, $v_{j}$ has only finitely many zeroes. Thus we have a well-defined algebraic intersection number int $\left(C, C^{\prime}\right)$. Recall from [6] that each intersection point contributes positively to int $\left(C, C^{\prime}\right)$ and intersection points persist under small perturbations.

Now suppose that $C, C^{\prime}$ in $\mathcal{M}_{\mathrm{w}}^{0}$ are neither identical nor disjoint, hence $\operatorname{int}\left(C, C^{\prime}\right)>0$. Pick a continuous path $\left(C_{\tau}\right)_{0 \leq \tau \leq 1}$ in $\mathcal{M}_{\mathrm{w}}^{0}$ with $C_{0}=C$ and $C_{1}=C^{\prime}$. For small $\tau$, we have $\operatorname{int}\left(C, C_{\tau}\right)=0$ by Theorem 2.7. We define

$$
\tau_{0}:=\inf \left\{0 \leq \tau \leq 1 \mid \operatorname{int}\left(C, C_{\tau}\right)>0\right\} \in(0,1) .
$$

Since intersections persist under perturbations, we must have $\operatorname{int}\left(C, C_{\tau_{0}}\right)=0$. Let $I_{\tau} \subset C$ be the (finite) set of intersections between $C$ and $C_{\tau}$ on the surface $C$. Now 
Vol. 80 (2005) The Weinstein conjecture for planar contact structures in dimension three 787

observe that for every neighborhood $U$ of the set of punctures on $C$ there is an $\varepsilon>0$ such that

$$
I_{\tau} \subset U \text { for all } 0<\tau<\tau_{0}+\varepsilon .
$$

For otherwise we would find a sequence $\tau_{n}>\tau_{0}$ with $\tau_{n} \rightarrow \tau_{0}$ and intersection points $z_{n} \in C \cap C_{\tau_{n}}$ with $z_{n} \notin U$. But then the $z_{n}$ would converge to an intersection point $z \in C \cap C_{\tau_{0}}$, contradicting $\operatorname{int}\left(C, C_{\tau_{0}}\right)=0$.

As above, write $C_{\tau}$ for $\tau>0$ near the $j$-th puncture as the graph of a section $v_{j}^{\tau}$ in the normal bundle to $C$ which approaches zero exponentially from the direction of an eigenfield $e_{j}^{\tau}$ of the asymptotic operator at $x_{j}$. Denote by wind $\left(e_{j}^{\tau}\right)$ the winding number of $e_{j}^{\tau}$ in a trivialization of the normal bundle, and by $\alpha_{j}$ the maximal winding number of an eigenvalue below the weight $\delta_{j}$. Define an integer valued function $i(\tau)$ by

$$
i(\tau)=\operatorname{int}\left(C, C_{\tau}\right)-\sum_{j=1}^{N}\left[\operatorname{wind}\left(v_{j}^{\tau}\right)-\alpha_{j}\right] .
$$

If $\tau$ is small the Implicit Function Theorem 2.7 yields wind $\left(v_{j}^{\tau}\right)=\alpha_{j}$ and $\operatorname{int}\left(C, C_{\tau}\right)=$ 0 , hence $i(\tau)=0$. We will show that $i(\tau)=0$ for all $\tau<\tau_{0}+\varepsilon$ for some $\varepsilon>0$. Since $\sum_{j=1}\left[\operatorname{wind}\left(v_{j}^{\tau}\right)-\alpha_{j}\right] \leq 0$, this then implies $\operatorname{int}\left(C, C_{\tau}\right) \leq 0$ for all $\tau<\tau_{0}+\varepsilon$, contradicting the choice of $\tau_{0}$.

Fix a sufficiently small neighbourhood $U=\cup_{j} U_{j}$ of the set of punctures on $C$. After trivializing the normal bundle of $C$, the restriction of each $v_{j}^{\tau}$ to $\bar{U}_{j}$ can be viewed as a complex valued function on $[0, \infty) \times S^{1}$ which satisfies a linear Cauchy Riemann type equation as in Lemma 2.5. Dividing $v_{j}^{\tau}$ by a suitable smooth positive function $\beta_{j}^{\tau}$ and compactifying the infinite half cylinder to $[0,1] \times S^{1}$, we obtain functions

$$
w_{j}^{\tau}:[0,1] \times S^{1} \longrightarrow \mathbb{C}
$$

with

$$
\operatorname{wind}\left(w_{j}^{\tau}(1, \cdot)\right)=\operatorname{wind}\left(e_{j}^{\tau}\right) .
$$

These functions are continuous in $\tau$ on the open half-cylinder $[0,1) \times S^{1}$. For $\varepsilon$ sufficiently small we have $I_{\tau} \subset U$ for all $0<\tau<\tau_{0}+\varepsilon$, hence $\operatorname{wind}\left(w_{j}^{\tau}(0, \cdot)\right)$ is well-defined and independent of $\tau \in\left(0, \tau_{0}+\varepsilon\right)$. As the zeros of $w_{j}^{\tau}$ correspond to the intersection points of $C$ and $C_{\tau}$ and their degrees equal the local intersection number, we have

$$
\operatorname{wind}\left(w_{j}^{\tau}(1, \cdot)\right)-\sum_{\left\{z: w_{j}^{\tau}(z)=0\right\}} \operatorname{deg}(z)=\operatorname{wind}\left(w_{j}^{\tau}(0, \cdot)\right) .
$$

We conclude that the left hand side is independent of $\tau \in\left(0, \tau_{0}+\varepsilon\right)$, and summing over $j$ yields $i(\tau)=0$ for all $\tau \in\left(0, \tau_{0}+\varepsilon\right)$. 


\section{Proof of the main result}

In this section we shall prove the main result, Theorem 1.6.

3.1. Finite energy foliations for Giroux forms. We start by explaining a result by Abbas [1] about turning leaves of an open book decomposition into solutions of equation (2.1).

Let us call two solutions $(S, j, \Gamma, \tilde{u})$ and $\left(S^{\prime}, j^{\prime}, \Gamma^{\prime}, \tilde{u}^{\prime}\right)$ of (2.1) equivalent if there exists a biholomorphic map $\phi:(S, j) \rightarrow\left(S^{\prime}, j^{\prime}\right)$ mapping $\Gamma$ to $\Gamma^{\prime}$ (preserving the enumeration) so that $\tilde{u}^{\prime} \circ \phi=\tilde{u}$. From now on a solution of our differential equation is an equivalence class $[S, j, \Gamma, \tilde{u}]$. Note that we have a natural $\mathbb{R}$-action on the solution set by associating to $c \in \mathbb{R}$ and $[S, j, \Gamma, \tilde{u}]$ the new solution

$$
c+[S, j, \Gamma, \tilde{u}]:=[S, j, \Gamma,(a+c, u)], \quad \tilde{u}=(a, u) .
$$

Given $[S, j, \Gamma, \tilde{u}]$, we denote by $C$ the image of $\tilde{u}$. Since all the maps $\tilde{u}$ of interest to us will be somewhere injective one can show that knowing $C$ we can reconstruct the underlying equivalence class $[S, j, \Gamma, \tilde{u}]$. A crucial concept for our discussion is the notion of a finite energy foliation $\mathcal{F}$.

Definition 3.1. A smooth foliation $\mathcal{F}$ of $\mathbb{R} \times M$ is called a finite energy foliation if every leaf $F$ is the image of an embedded solution $[S, j, \Gamma, \tilde{u}]$ of $(2.1)$,

$$
F=\tilde{u}(\dot{S}),
$$

and with every leaf $F \in \mathcal{F}$ also $c+F \in \mathcal{F}$ for every $c \in \mathbb{R}$, i.e., the foliation is $\mathbb{R}$-invariant.

Finite energy foliations are known to be a useful tool in studying the dynamics of Reeb vector fields as well as topological applications, see [18], [16], [13]. The following theorem is proved by Abbas in [1].

Theorem 3.2. Assume that $M$ is a closed three-manifold equipped with a planar contact structure $\xi$ and a Giroux form $\lambda_{\text {Giroux }}$ with nondegenerate closed Reeb orbits. Let $J$ be any compatible complex multiplication on $\xi$. Then there exist an open book decomposition ( $L$, pr) for $\xi$ with Giroux form $\lambda_{\text {Giroux }}$ and a finite energy foliation $\mathcal{F}$ of $\mathbb{R} \times M$ with the following properties.

- The cylinders over the binding orbits in $L$ are leaves of $\mathcal{F}$, called the trivial leaves.

- Every nontrivial leaf is the image of a finite energy sphere with only positive punctures. These punctures are in 1-1 correspondence with, and asymptotic to, the binding orbits. 
Vol. 80 (2005) The Weinstein conjecture for planar contact structures in dimension three 789

- The projection to $M$ of any nontrivial leaf can be compactified (by the binding orbits) to a page of the open book decomposition ( $L, \mathrm{pr})$.

Remark 3.3. (1) The open book decomposition ( $L$, pr) in the theorem may differ from the planar open book decomposition we started with.

(2) Theorem 3.2 can be proved along the following lines, using the compactness theorem for symplectic field theory [2].

The first step consists of modifying a leaf $u_{0}$ of the given planar open book decomposition $\left(L_{0}, \mathrm{pr}_{0}\right)$ near its punctures so that there is a suitable function $a_{0}$ such that $\tilde{u}_{0}=\left(a_{0}, u_{0}\right)$ solves the differential equation near the punctures. This is achieved by choosing $J: \xi \rightarrow \xi$ near the binding $L_{0}$ and the complex structure $j$ near the punctures in a very special way so that solutions can be written down explicitly. We then look for a global solution $\tilde{u}=(a, u)$ to the equation $u^{*} \lambda \circ j=d a$ of the form $u=\phi_{f}\left(u_{0}\right)$, where $f$ is a suitable real valued function on the closed surface $S$ (a sphere in our case) and where $\phi_{t}$ denotes the flow of the Reeb vector field. This amounts to solving an inhomogeneous Cauchy Riemann equation for the function $a-a_{0}+i f$ on the sphere, which is possible because on the sphere $\bar{\partial}$ is surjective. The first part of (2.1) involving $\pi \circ T u$ can then be used to define a $z$-dependent complex structure $J^{+}$on $\xi$ so that

$$
\pi T u(z) \circ j=J^{+}(z, u(z)) \circ \pi T u(z) .
$$

A cobordism argument similar to the one in Section 3 of this paper can then be used to deform the parameter dependent complex structure $J^{+}$into one which does not depend explicitly on $z$, say $J^{-}$. We pick a complex structure $\tilde{J}=\tilde{J}(z, a, u)$, $(z, a, u) \in S \times \mathbb{R} \times M$, on $\mathbb{R} \times M$ such that $\tilde{J} \equiv \tilde{J}^{+}$for $a \geq 1$ and $\tilde{J} \equiv \tilde{J}^{-}$for $a \leq 0$, and we study the corresponding PDE (2.2). There is an implicit function theorem and the compactness result [2] can be applied. Assume that $\tilde{u}_{k}=\left(a_{k}, u_{k}\right)$ is a sequence of solutions such that

$$
\inf a_{k} \rightarrow r \in \mathbb{R} \text {. }
$$

Although there is no statement corresponding to Theorem 3.4 in this paper, a solution where the infimum equals $r$ can still be found (the part in the cobordism of the broken punctured holomorphic curve in the limit). An argument similar to the one in Section 3.4 of this paper produces a finite energy solution $\tilde{u}=(a, u)$ to the PDE in the negative part $\left(\mathbb{R} \times M, \tilde{J}^{-}\right)$with only positive punctures such that $u$ is an embedding transverse to the Reeb vector field. The collection $L$ of positive punctures of $u$ may differ from the binding $L_{0}$ of the original open book decomposition. It is then shown that there is a compact 1-dimensional family of such solutions which form an open book decomposition with binding $L$.

We will refer to the nontrivial leaves in Theorem 3.2 as Abbas solutions. 
3.2. A cobordism. Suppose now that $\xi$ is supported by a planar open book decomposition. Let $\lambda_{\text {Giroux }}$ be an associated Giroux form with nondegenerate elliptic binding orbits. We are interested in the Reeb flow of a different contact form $\lambda$ defining $\xi$. Multiplying the Giroux form by some positive constant, we may assume that

$$
\lambda_{\text {Giroux }}=f^{+} \cdot \lambda
$$

for a function $f^{+}>1$ on $M$. Pick $R>0$ and $\tilde{J}$ as in the beginning of Section 2 (with $\lambda^{+}=\lambda_{\text {Giroux }}$ and $\lambda^{-}=\lambda$ ) and consider the PDE (2.2) for generalized finite energy spheres. Observe that any Abbas solution is, after translating it by a sufficiently large positive constant, a solution of (2.2). Denote by $\mathcal{A}$ the collection of all images of Abbas solutions which are contained in $[R, \infty) \times M$. Of course, any two such solutions are either disjoint or identical, and the space $\mathcal{A}$ is connected.

Let $A=\tilde{u}(\dot{S})$ be an Abbas solution. Pick weights $\delta_{j}$ as in Lemma 2.6 so that $A$ has weighted Fredholm index $\operatorname{ind}_{\mathrm{w}}(A)=2$. Denote by $\mathcal{M}_{\mathrm{w}}$ the space of solutions of equation (2.2) with positive punctures asymptotic to the binding orbits of $\lambda_{\text {Giroux }}$ and with weights $\delta_{j}$. Note that since all Abbas solutions have the same winding numbers at the punctures, we have $\mathcal{A} \subset \mathcal{M}_{\mathrm{w}}$. Let $\mathcal{M}_{\mathrm{w}}^{0}$ be the connected component of $\mathcal{M}_{\mathrm{w}}$ containing $\mathcal{A}$.

3.3. A compactness statement. Assume for the moment that $\lambda$ is nondegenerate. Then we have the following compactness result for $\mathcal{M}_{\mathrm{w}}^{0}$.

Theorem 3.4. Assume that $\lambda$ is nondegenerate. Let $C_{k}=\tilde{u}_{k}(\dot{S})$ be a sequence in $\mathcal{M}_{\mathrm{w}}^{0}$ so that a $\left(C_{k}\right) \rightarrow r \in \mathbb{R}$. Then, after passing to a subsequence, there exists an element $C \in \mathcal{M}_{\mathrm{w}}^{0}$ so that for suitable parametrizations $\tilde{u}_{k} \rightarrow \tilde{u}$ in $C_{\mathrm{loc}}^{\infty}$. Moreover, $a(C)=\lim _{k \rightarrow \infty} a\left(C_{k}\right)$.

Proof. We apply the compactness theorem for symplectic field theory [2]. After passing to a subsequence, the $C_{k}$ converge to a broken punctured holomorphic curve of type $\left(k^{-}\left|k^{0}\right| k^{+}\right)$. This means that the limit curve has $k^{+} \geq 0$ components in the symplectization $\left(\mathbb{R} \times M, \tilde{J}^{+}\right)$of the positive end, $k^{-} \geq 0$ components in the symplectization $\left(\mathbb{R} \times M, \tilde{J}^{-}\right)$of the negative end, and $k^{0} \in\{0,1\}$ components in the cobordism $(\mathbb{R} \times M, \tilde{J})$. From $a\left(C_{k}\right) \rightarrow r \in \mathbb{R}$ we conclude that $k^{-}=0$ and $k^{0}=1$, so the limit curve is of type $\left(0|1| k^{+}\right)$. If $k^{+}=0$ the assertion of the proposition follows from the definition of convergence in [2]. Thus suppose that $k^{+} \geq 1$. Then the top layer is a (not necessarily connected) curve solving the homogeneous $\tilde{J}^{+}$-problem. By the stability requirement in [2], this layer contains at least one component $\hat{C}$ that is not a cylinder over a closed Reeb orbit. Let $\hat{u}$ be a parametrization of $\hat{C}$. Note that $\hat{u}$ must be somewhere injective since the positive asymptotic limits are simplycovered (they are binding orbits of the open book decomposition). Therefore, $\hat{u}$ is not a branched covering of a cylinder over a closed Reeb orbit, and hence $\hat{C}$ has to 
Vol. 80 (2005) The Weinstein conjecture for planar contact structures in dimension three 791

intersect a nontrivial leaf of the Abbas foliation. On the other hand, $\hat{C}$ cannot be identical to such a leaf since it has at least one negative puncture. From the definition of convergence and positivity of intersections it follows that there is a sequence of Abbas solutions $A_{k} \in \mathcal{A}$ such that $C_{k} \cap A_{k} \neq \emptyset$ and $C_{k} \neq A_{k}$ for large $k$. Since $\mathcal{A} \subset \mathcal{M}_{\mathrm{w}}^{0}$, this contradicts Proposition 2.10.

3.4. Conclusion. The planar Weinstein conjecture is now proved as follows. We keep assuming that $\lambda$ is nondegenerate. First note that

$$
\inf _{C \in \mathcal{M}_{\mathrm{w}}^{0}} a(C)=-\infty
$$

Indeed, arguing indirectly, suppose the left-hand side defines a real number $r$. Take a sequence $C_{k} \in \mathcal{M}_{\mathrm{w}}^{0}$ with $a\left(C_{k}\right) \rightarrow r$. By Theorem 3.4, after taking a subsequence, we find a $C \in \mathcal{M}_{\mathrm{w}}^{0}$ with

$$
a(C)=\lim _{k \rightarrow \infty} a\left(C_{k}\right)=\inf _{C^{\prime} \in \mathcal{M}_{\mathrm{w}}^{0}} a\left(C^{\prime}\right) .
$$

By Corollary 2.9, there exists a $C^{\prime} \in \mathcal{M}_{\mathrm{w}}^{0}$ with $a\left(C^{\prime}\right)<a(C)$, giving a contradiction. The proof is now completed by taking a sequence $C_{k} \in \mathcal{M}_{\mathrm{w}}^{0}$ with

$$
a\left(C_{k}\right) \rightarrow-\infty
$$

We apply again the compactness theorem for symplectic field theory [2]. After passing to a subsequence, the $C_{k}$ converge to a broken punctured holomorphic curve of type $\left(k_{-}\left|k^{0}\right| k_{+}\right)$. From $a\left(C_{k}\right) \rightarrow-\infty$ we conclude $k_{-} \geq 1$ (and consequently $k^{0}=1$ ). By the definition of convergence in [2], the lowest layer of the limit curve contains a non-constant (special) finite energy sphere $\hat{C}$ for $\left(M, \lambda, J^{-}\right)$having only positive punctures. At the punctures, $\hat{C}$ is asymptotic to periodic Reeb orbits $x_{j}$ of the contact form $\lambda$. By construction, their homology classes $\left[x_{j}\right]$ satisfy $\sum\left[x_{j}\right]=0$, where we sum over all the punctures. This proves Theorem 1.6 in the case that $\lambda$ is nondegenerate.

If $\lambda$ is degenerate we can take a sequence $f^{(k)}: M \rightarrow(0, \infty)$ of smooth functions converging in $C^{\infty}$ to the constant function $f(x) \equiv 1$ so that the contact forms $f^{(k)} \lambda$ are nondegenerate. By the result in the nondegenerate case, we find for every $k$ a finite set of periodic Reeb orbits $x_{j}^{(k)}$ for $f^{(k)} \lambda$ whose homology classes sum up to zero. By the proof of the compactness theorem in [2], the number of orbits for each $k$ is bounded by a constant independent of $k$. So after passing to a subsequence, we may assume that their number is constant. Then by the Arzela-Ascoli theorem, after passing to a subsequence, the $x_{j}^{(k)}$ converge in $C^{\infty}$ as $k \rightarrow \infty$ to periodic Reeb orbits for the contact form $\lambda$. Clearly, the homology classes of the $x_{j}$ sum up to zero. This concludes the proof of Theorem 1.6. 


\section{References}

[1] C. Abbas, Holomorphic open book decompositions. In preparation.

[2] F. Bourgeois, Y. Eliashberg, H. Hofer, K. Wysocki and E. Zehnder, Compactness results in symplectic field theory. Geom. Topol. 7 (2003), 799-888 (electronic). Zbl 02062477 MR 2026549

[3] D. L. Dragnev, Fredholm Theory and Transversality for noncompact pseudoholomorphic maps in symplectizations. Comm. Pure Appl. Math. 57 (2004), 726-763. Zbl 02081808 MR 2038115

[4] Y. Eliashberg, A few remarks about symplectic filling. Geom. Topol. 8 (2004), 277-293 (electronic). Zbl 02105189 MR 2023278

[5] J. Etnyre, Planar open book decompositions and contact strucures. Int. Math. Res. Not. 79 (2004), 4255-4267. Zbl 02151805 MR 2126827

[6] A. Floer, H. Hofer and D. Salamon, Transversality in elliptic Morse theory for the symplectic action. Duke Math. J. 80 (1995), 251-292. Zbl 0846.58025 MR 1360618

[7] E. Giroux, Lecture given at the Georgia Topology conference, May 24, 2001. Notes available at http://www.math.uga.edu/ topology/2001/giroux.pdf

[8] E. Giroux, Géométrie de contact: de la dimension trois vers les dimensions supérieures. In Proceedings of the ICM Beijing 2002, Vol. II, Higher Education Press, Beijing 2002, 405-414. Zbl 1015.53049 MR 1957051

[9] H. Hofer, Pseudoholomorphic curves in symplectizations with applications to the Weinstein conjecture in dimension three. Invent. Math. 114 (1993), 515-563. Zbl 0797.58023 MR 1244912

[10] H. Hofer, Holomorphic curves and real three-dimensional dynamics. In GAFA 2000 (Tel Aviv, 1999), Geom. Funct. Anal., Special Volume, Part II, 2000, 674-704. Zbl 01643844 MR 1826268

[11] H. Hofer and M. Kriener, Holomorphic curves in contact dynamics. In Differential equations: La Pietra 1996, Proc. Sympos. Pure Math. 65, Amer. Math. Soc., Providence, RI, 1999, 77-131. Zbl 0931.37026 MR 1662750

[12] H. Hofer, K. Wysocki and E. Zehnder, Properties of pseudo-holomorphic curves in symplectisations II: Embedding controls and algebraic invariants. Geom. Funct. Anal. 5 (1995), 270-328. Zbl 0845.57027 MR 1334869

[13] H. Hofer, K. Wysocki and E. Zehnder, A characterization of the tight three-sphere. Duke Math. J. 81 (1995), 159-226; Correction to "A characterisation of the tight threesphere”. Duke Math. J. 89 (1997), 603-617.Zbl 0861.57026 MR 1381975 Zbl 0903.57009 MR 1470344

[14] H. Hofer, K. Wysocki and E. Zehnder, Properties of pseudoholomorphic curves in symplectisations I: Asymptotics. Ann. Inst. H. Poincaré Anal. Non Linéaire 13 (1996), 337-379. Zbl 0861.58018 MR 1395676

[15] H. Hofer, K. Wysocki and E. Zehnder, Properties of pseudoholomorphic curves in symplectisations IV: Asymptotics with degeneracies. In Contact and Symplectic Geometry, Publ. Newton Inst. 8, Cambridge University Press, Cambridge 1996, 78-117. Zbl 0868.53043 MR 1432460 
Vol. 80 (2005) The Weinstein conjecture for planar contact structures in dimension three 793

[16] H. Hofer, K. Wysocki and E. Zehnder, The dynamics on strictly convex energy surfaces in $\mathbb{R}^{4}$. Ann. Math. (2) 148 (1998), 197-289. Zbl 0944.37031 MR 1652928

[17] H. Hofer, K. Wysocki and E. Zehnder, Properties of pseudoholomorphic curves in symplectisations III: Fredholm theory. In Topics in Nonlinear Analysis, Progr. Nonlinear Differential Equations Appl. 35, Birkhäuser, Basel 1999, 381-475. Zbl 0924.58003 MR 1725579

[18] H. Hofer, K. Wysocki and E. Zehnder, Finite energy foliations of tight three-spheres and Hamiltonian dynamics. Ann. Math. (2) 157 (2003), 125-255. Zbl 01933157 MR 1954266

[19] D. Rolfsen, Knots and Links. Mathematics Lecture Series 7, Publish or Perish Inc., Berkeley, CA, 1976. Zbl 0339.55004 MR 0515288

[20] A. Weinstein, On the hypotheses of Rabinowitz's periodic orbit theorems. J. Differential Equations 33 (1979), 353-358. Zbl 0388.58020 MR 0543704

Received June 29, 2004

Casim Abbas, Department of Mathematics Michigan State University East Lansing, MI 48824, U.S.A.

E-mail: abbas@math.msu.edu

Kai Cieliebak, Mathematisches Institut, Ludwig-Maximilians-Universität, Theresienstr.39, D-80333 München, Germany

E-mail: kai@mathematik.uni-muenchen.de

Helmut Hofer, Courant Institute, New York University, 251 Mercer Street, New York, NY 10012, U.S.A.

E-mail: hofer@cims.nyu.edu 